\title{
The Prevalence of Cardiac Risk Factors in Men with Localized Prostate Cancer Undergoing Androgen Deprivation Therapy in British Columbia, Canada
}

\author{
Margot K. Davis, ${ }^{1}$ Jennifer L. Rajala, ${ }^{2}$ Scott Tyldesley, ${ }^{3}$ Tom Pickles, ${ }^{3}$ and Sean A. Virani ${ }^{1}$ \\ ${ }^{1}$ Division of Cardiology, University of British Columbia, Vancouver, BC, Canada V5Z 1M9 \\ ${ }^{2}$ Division of Cardiology, Royal Jubilee Hospital, Victoria, BC, Canada V8R $1 J 8$ \\ ${ }^{3}$ Department of Radiation Oncology, British Columbia Cancer Agency, Vancouver, BC, Canada V5Z 4E6
}

Correspondence should be addressed to Sean A. Virani; svirani@telus.net

Received 5 September 2014; Revised 8 December 2014; Accepted 9 December 2014

Academic Editor: Daniel Lenihan

Copyright (C) 2015 Margot K. Davis et al. This is an open access article distributed under the Creative Commons Attribution License, which permits unrestricted use, distribution, and reproduction in any medium, provided the original work is properly cited.

\begin{abstract}
Background. While androgen deprivation therapy (ADT) reduces the risk of prostate cancer-specific mortality in high-risk localized prostate cancer, it adversely affects cardiovascular (CV) risk factor profiles in treated men. Methods. We retrospectively reviewed the charts of 100 consecutive men with intermediate- or high-risk localized prostate cancer referred to the British Columbia Cancer Agency for ADT. Data on CV risk factors and disease were collected and Framingham risk scores were calculated. Results. The median age of the study cohort was 73 years. Established cardiovascular disease was present in $25 \%$ of patients. Among patients without established CV disease, calculated Framingham risk was high in 65\%, intermediate in 33\%, and low in $1 \%$. Baseline hypertension was present in $58 \%$ of patients, dyslipidemia in $51 \%$, and diabetes or impaired glucose tolerance in $24 \%$. Hypertension was more prevalent in the study cohort than in an age- and sex-matched population sample (OR 1.74, $P=0.006)$; diabetes had a similar prevalence (OR 0.93, $P=0.8$ ). Conclusions. Patients receiving ADT have a high prevalence of cardiovascular disease and risk factors and are more likely to be hypertensive than population controls. Low rates of CV risk screening suggest opportunities for improved primary and secondary prevention of $\mathrm{CV}$ disease in this population.
\end{abstract}

\section{Introduction}

Prostate cancer is the most common cancer diagnosed in men, affecting 1 in 7 men in North America [1,2]. Androgen deprivation therapy (ADT) with gonadotropin releasing hormone $(\mathrm{GnRH})$ agonists has been a mainstay of therapy for locally advanced and metastatic prostate cancer since the 1990s and is also increasingly used in the neoadjuvant setting prior to radiotherapy for early disease [3].

While ADT decreases the risk of prostate cancer-specific mortality in advanced prostate cancer, it is associated with a number of adverse metabolic effects. The risk of developing diabetes while on ADT increases by up to $44 \%[4,5]$ as lean body mass is significantly reduced and replaced by increased fat mass $[6,7]$. Several large population-based studies have indicated that men receiving ADT are at increased risk of fatal and nonfatal cardiovascular events $[5,8,9]$. Moreover, men with cardiovascular risk factors or previous cardiovascular events are at particularly high risk $[9,10]$.

No specific guidelines exist for the screening of patients with prostate cancer who have preexisting cardiovascular disease or who may be at risk for cardiovascular morbidity and mortality as a result of ADT. Studies have shown that cancer patients and survivors may be less likely to receive therapies directed at cardiovascular risk factor modification compared to other patients [11]. Underrecognition of risk factors and subsequent undertreatment may represent an important care gap in survivor populations. As such, appropriate treatment and modification of cardiovascular risk factors may minimize treatment related adverse effects. With this in mind, we investigated the burden of cardiovascular risk among patients receiving ADT for intermediate- and high-risk localized prostate cancer at our centre and described the measures taken to risk-stratify these patients prior to therapy. 


\section{Methods}

2.1. Patient Population and Treatment. The study population included 100 consecutive men with intermediate- or high-risk prostate cancer who were referred to the British Columbia Cancer Agency (BCCA) between October 1, 2011, and October 31, 2012, and who were treated with combined ADT and radiotherapy with curative intent. Patients were included if they had been referred to an oncologist within 3 months of cancer diagnosis and if their treatment plan included curative intent radiotherapy and $\geq 6$ months of ADT. Patients were excluded if they had metastatic prostate cancer. Radiotherapy protocols and choices of ADT regimens were at the discretion of treating physicians.

2.2. Data Collection. Data was collected by retrospective chart review. Data on patient demographics, past medical history, prior cardiac history, and medications were collected from the oncology chart. If a specific CV risk factor was not mentioned in the past medical history, the patient was presumed not to have that risk factor unless (1) the patient was on antihypertensive medications, in which case they were considered to have hypertension, or (2) the patient was on a statin medication or their lipid profile revealed an LDL $\geq 3.5 \mathrm{mmol} / \mathrm{L}$ or a non-HDL-C of $\geq 4.3 \mathrm{mmol} / \mathrm{L}$, in which case they were considered to have dyslipidemia. Systolic blood pressure was recorded from the initial consult with the oncologist or from the anaesthesia before operative consult or other specialist consults if not available on the first visit. The electronic chart and the common provincial laboratory system were accessed to find any lipid profiles from the year prior to or concomitant with the start of ADT. Lastly, the presence of any CV investigations on the chart, either in the year prior to the initiation of ADT or in response to the start of ADT, as well as any referral to a cardiology or internal medicine service for cardiac assessment was recorded. The University of British Columbia Research Ethics Board approved the data collection protocol used in this study.

2.3. Risk Calculation. A Framingham risk score (FRS) [12] was calculated on all patients who did not have underlying coronary heart, cerebrovascular, or peripheral arterial disease (PAD) to estimate the 10 -year risk of CV events. A modified Charlson Comorbidity Index [13] was calculated on all patients to estimate 10 -year mortality risk. Prostate cancer diagnosis was not accorded any points in the modified Charlson score. If no blood pressure was recorded on the chart, a score of 0 was used for hypertension in the Framingham risk calculation. Because ADT is known to increase serum lipids, any lipid measurements taken after the start of ADT were not used in the Framingham risk calculation. If no lipid profile was available, a score of 0 was used for the HDL and total cholesterol values in calculating the FRS.

2.4. Comparison Cohort. The prevalence of two cardiovascular risk factors, hypertension and diabetes, was compared to values reported for males aged 65 years and over in British Columbia in the 2011-2012 Canadian Community
Health Survey, administered by Statistics Canada [14]. These population prevalence values refer to the proportion of the population who reported that they had been diagnosed by a health professional as "having high blood pressure" or "having type 1 or type 2 diabetes," respectively.

2.5. Statistical Analysis. Proportions of men in the prostate cancer cohort and comparison cohort with hypertension and diabetes were compared using Chi-square tests. $P$ values $<0.05$ were considered significant. Statistical analysis was performed using Microsoft Excel for Mac Version 14.4.6 (Microsoft Corporation, Redmond, WA).

\section{Results}

Baseline demographics for the study cohort are displayed in Table 1 . The median age of the cohort was 73 years (range $50-87$ years). Thirty (30\%) subjects had moderately differentiated cancer (Gleason score 5-7) and 70 (70\%) had poorly differentiated disease (Gleason score 8-10). The median initial PSA value was $12 \mathrm{ng} / \mathrm{mL}$ (IQR 8.2, 21.1). No patient had any evidence of metastatic prostate cancer on either bone scan or staging CT scan. Most patients (82\%) had a modified Charlson Comorbidity Index of 0 .

3.1. Baseline Cardiovascular Risk. Previously documented coronary artery disease (CAD) was present in $17 \%$ of patients, with 9 of these patients having had a past percutaneous coronary intervention (PCI) and 5 patients having undergone coronary artery bypass grafting (CABG) surgery. A history of stroke was present in 7\%, and $5 \%$ had a history of PAD. A total of 25 patients were excluded from the Framingham risk calculation due to previous history of CAD, PAD, or stroke. A history of cardiac arrhythmia was documented in $10 \%$ of patients, 5 of whom had atrial fibrillation. The presence of baseline cardiac risk factors was common in this cohort (Table 2 ), with $58 \%$ of patients having a history of hypertension, $51 \%$ having a history of dyslipidemia, and $17 \%$ of patients having a history of diabetes. Only $4 \%$ of patients had no cardiac risk factors at all. Complete data to calculate a Framingham risk score was present for only $17 \%$ of patients; $62 \%$ of those studied did not have a lipid profile in the year prior to starting ADT and 58\% did not have a blood pressure recording on the chart. Despite amending the Framingham risk calculation with scores of 0 for the missing data, only 1 patient was in the low risk Framingham category. Most patients, $69 \%$, were in the high Framingham risk category and $30 \%$ were calculated to be at moderate Framingham risk.

3.2. Cardiovascular Investigations. Only $35 \%$ of patients had an ECG present on the chart (Figure 1). 22 of 35 ECGs were classified as normal. The most common abnormalities noted on ECG were nonspecific ST segment changes $(n=5)$, followed by intraventricular conduction delays or bundle branch block $(n=3)$. Less common was evidence of a prior myocardial infarct $(n=2)$ or left ventricular hypertrophy $(n=2)$. Only $6 \%$ of the patients studied had further testing for cardiac ischemia with either exercise treadmill testing or 
TABLE 1: Baseline characteristics of 100 men referred for androgen deprivation therapy.

\begin{tabular}{|c|c|}
\hline Age at diagnosis & $73(50-87)$ \\
\hline Vascular disease & $25(25 \%)$ \\
\hline Coronary artery disease & $17(17 \%)$ \\
\hline Percutaneous coronary intervention & $9(9 \%)$ \\
\hline Coronary artery bypass surgery & $5(5 \%)$ \\
\hline Stroke & $7(7 \%)$ \\
\hline Peripheral arterial disease & $5(5 \%)$ \\
\hline Atrial fibrillation & $5(5 \%)$ \\
\hline Supraventricular tachycardia & $1(1 \%)$ \\
\hline Arrhythmia, not specified & $4(4 \%)$ \\
\hline Cardiac pacemaker & $1(1 \%)$ \\
\hline Pericarditis & $1(1 \%)$ \\
\hline Coronary vasospasm & $1(1 \%)$ \\
\hline History of heart failure & $1(1 \%)$ \\
\hline \multicolumn{2}{|l|}{ Framingham risk category } \\
\hline High risk & $49(65 \%)^{*}$ \\
\hline Intermediate risk & $25(33 \%)^{*}$ \\
\hline Low risk & $1(1 \%)^{*}$ \\
\hline \multicolumn{2}{|l|}{ Gleason score } \\
\hline Moderately differentiated (5-7) & $30(30 \%)$ \\
\hline Poorly differentiated (8-10) & $70(70 \%)$ \\
\hline \multicolumn{2}{|l|}{ Clinical stage } \\
\hline $\mathrm{T} 1$ & $27(27 \%)$ \\
\hline $\mathrm{T} 2$ & $43(43 \%)$ \\
\hline T3 & $23(23 \%)$ \\
\hline $\mathrm{T} 4$ & $3(3 \%)$ \\
\hline $\mathrm{X}$ & $4(4 \%)$ \\
\hline \multicolumn{2}{|l|}{ Initial PSA (ng/mL) } \\
\hline$<5$ & $9(9 \%)$ \\
\hline $5-10$ & $29(29 \%)$ \\
\hline$>10$ & $62(62 \%)$ \\
\hline \multicolumn{2}{|l|}{ Updated Charlson Comorbidity Index } \\
\hline 0 & $82(82 \%)$ \\
\hline 1 & $14(14 \%)$ \\
\hline$\geq 2$ & $4(4 \%)$ \\
\hline \multicolumn{2}{|l|}{ Hormonal treatment used } \\
\hline Goserelin & $72(72 \%)$ \\
\hline Leuprolide & $25(25 \%)$ \\
\hline Degarelix & $2(2 \%)$ \\
\hline Bicalutamide & $92(92 \%)$ \\
\hline Buserelin & $1(1 \%)$ \\
\hline Flutamide & $1(1 \%)$ \\
\hline
\end{tabular}

Expressed as median (range) or number (percentage).

${ }^{*}$ Expressed as percentage of patients without baseline history of vascular disease.

myocardial perfusion imaging (Table 3). Documentation of a previous cardiology assessment was present for 6 patients, while 3 more patients were referred to a cardiology or internal medicine service for further work-up after oncology consult.

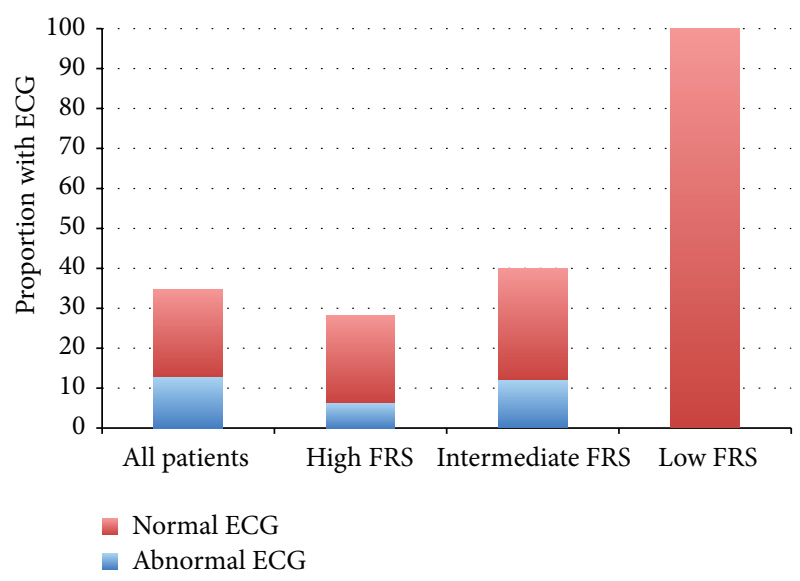

FIGURE 1: Proportions of patients referred for androgen deprivation therapy with normal and abnormal electrocardiograms available for review. ECG, electrocardiogram; FRS, Framingham risk score.

3.3. Comparison with Population Prevalence Values. In the 2011-2012 Canadian Community Health Survey, 44.3\% $(n=$ 139, 502) of men aged 65 years and over in British Columbia reported that they had been diagnosed with high blood pressure, and $18.0 \%(n=56,540)$ reported that they had been diagnosed with diabetes. Compared to the population sample, the odds ratio for having hypertension in our prostate cancer cohort was $1.74(P=0.006)$ and the odds ratio for having diabetes was $0.93(P=0.8)$.

\section{Discussion}

In this cross-sectional study of cardiovascular risk profiles among men referred for management of localized prostate cancer with ADT, we identified high prevalences of baseline cardiovascular risk factors and of cardiovascular disease. In addition to the $25 \%$ of men with established cardiovascular disease in our study population, at least $69 \%$ of subjects without established disease had a high FRS which is associated with a $\geq 20 \% 10$-year risk of developing coronary heart disease, prior to initiation of ADT. By contrast, our cohort had a low prevalence of other comorbidities and a low expected mortality predicted by the Charlson Comorbidity Index.

We identified a higher prevalence of hypertension among our cohort than was reported among men aged 65 years and over in the same geographic area during the 2011-2012 Canadian Community Health Survey, suggesting that our prostate cancer population may be at higher risk of cardiovascular disease than members of the general population without prostate cancer, even after controlling for age and sex. The reasons for this association are not clear, but we can hypothesize that men with prostate cancer may have an increased burden of cardiovascular risk factors because many of these risk factors are also associated with risk of cancer. Indeed, previous studies have demonstrated increased prostate cancer risk among men with risk factors such as hypertension [15, 16], dyslipidemia [17], and the metabolic syndrome $[15,17,18]$. Increased prostate cancer risk has also 
TABLE 2: Cardiac risk factors present in 100 men prior to receiving androgen deprivation therapy.

\begin{tabular}{|c|c|c|c|c|}
\hline Risk factor & $\begin{array}{c}\text { All patients } \\
(n=100)\end{array}$ & $\begin{array}{c}\text { High FRS } \\
(n=49) \\
\end{array}$ & $\begin{array}{l}\text { Intermediate } \\
\text { FRS }(n=25)\end{array}$ & $\begin{array}{c}\text { Low FRS } \\
(n=1)\end{array}$ \\
\hline Hypertension & $58(58 \%)$ & $28(57 \%)$ & $7(28 \%)$ & 0 \\
\hline Diabetes mellitus or IGT & $22(22 \%)$ & $11(22 \%)$ & $6(24 \%)$ & 0 \\
\hline \multicolumn{5}{|l|}{ Cigarette smoking } \\
\hline Never & $46(46 \%)$ & $22(45 \%)$ & $14(56 \%)$ & $1(1 \%)$ \\
\hline Current & $6(6 \%)$ & $3(6 \%)$ & $2(8 \%)$ & 0 \\
\hline Quit $<5$ years ago & $3(3 \%)$ & $1(2 \%)$ & $1(4 \%)$ & 0 \\
\hline Quit > 5 years ago & $44(44 \%)$ & $23(47 \%)$ & $7(28 \%)$ & 0 \\
\hline Family history of CAD & $10(10 \%)$ & $2(4 \%)$ & 0 & 0 \\
\hline Dyslipidemia & $51(51 \%)$ & $19(39 \%)$ & $10(40 \%)$ & 0 \\
\hline Chronic kidney disease $\left(\mathrm{GFR}<60 \mathrm{~mL} / \mathrm{min} / 1.73 \mathrm{~m}^{2}\right)$ & $10(10 \%)$ & $6(12 \%)$ & $1(4 \%)$ & 0 \\
\hline \multicolumn{5}{|l|}{ Medication class } \\
\hline ASA & $33(33 \%)$ & $12(24 \%)$ & $2(8 \%)$ & 0 \\
\hline Clopidogrel & $1(1 \%)$ & 0 & 0 & 0 \\
\hline Warfarin & $3(3 \%)$ & $3(6 \%)$ & 0 & 0 \\
\hline ACE inhibitor & $32(32 \%)$ & $16(33 \%)$ & $2(8 \%)$ & 0 \\
\hline $\mathrm{ARB}$ & $16(16 \%)$ & $8(16 \%)$ & $2(8 \%)$ & 0 \\
\hline Beta blocker & $17(17 \%)$ & $7(14 \%)$ & $1(4 \%)$ & 0 \\
\hline Other antihypertensives & $31(31 \%)$ & $15(31 \%)$ & $4(16 \%)$ & 0 \\
\hline Statin & $40(40 \%)$ & $13(27 \%)$ & $6(24 \%)$ & 0 \\
\hline Nitroglycerin & $1(1 \%)$ & 0 & 0 & 0 \\
\hline Insulin & $4(4 \%)$ & $3(6 \%)$ & 0 & 0 \\
\hline
\end{tabular}

ACE: angiotensin converting enzyme; ARB: angiotensin receptor blocker; ASA: acetylsalicylic acid; CAD: coronary artery disease; FRS: Framingham risk score; GFR: glomerular filtration rate; IGT: impaired glucose tolerance.

TABLE 3: Cardiac evaluations performed in men with prostate cancer referred for androgen deprivation therapy.

\begin{tabular}{lcccc}
\hline Investigation & $\begin{array}{c}\text { All patients } \\
(n=100)\end{array}$ & $\begin{array}{c}\text { High FRS } \\
(n=49)\end{array}$ & $\begin{array}{c}\text { Intermediate } \\
\text { FRS }(n=25)\end{array}$ & $\begin{array}{c}\text { Low FRS } \\
(n=1)\end{array}$ \\
\hline Echocardiogram & 3 & $1(2 \%)$ & $1(4 \%)$ & 1 \\
Exercise treadmill test & 3 & 1 & $1^{\dagger}$ & 0 \\
$\quad$ Positive for ischemia & 1 & 0 & 0 & 0 \\
$\quad$ Negative for ischemia & 2 & 1 & 0 & 0 \\
Myocardial perfusion imaging & 2 & 0 & 0 & 0 \\
Positive for ischemia & 1 & 0 & 1 & 0 \\
Negative for ischemia & 1 & 0 & 0 & 0 \\
Stress echocardiogram & 1 & 0 & $1^{\dagger}$ & 0 \\
Positive for ischemia & 0 & 0 & 0 & 0 \\
Negative for ischemia & 1 & 1 & 0 & 0 \\
Holter monitor & 1 & 1 & 0 \\
Normal & 1 & 0 & 0 \\
Abnormal & 0 & 0 & 0 \\
\hline
\end{tabular}

FRS: Framingham risk score.

${ }^{\dagger}$ One patient had evidence of ischemia on exercise treadmill testing and was subsequently referred for stress echocardiogram which showed no ischemia.

been observed among men with established coronary artery disease [19]. Men with metabolic syndrome and hypertension are also at increased risk of biochemical recurrence following radical prostatectomy for prostate cancer [20-22]. Although the pathways by which the metabolic syndrome and its components increase cancer risk have not been fully elucidated, likely mechanisms involve insulin resistance, hyperinsulinemia, and elevated levels of insulin-like growth factor (IGF)-1, as well as increased levels of inflammation [17]. It is, therefore, somewhat surprising that diabetes, a state 
associated with insulin resistance, was not more prevalent in our cohort than in the general population. However, this finding was consistent with previous studies and it has been suggested that this may be because advanced diabetes is associated with low insulin levels due to pancreatic $\beta$-cell failure, resulting in a late protective effect [21].

ADT has been associated with worsening cardiovascular risk profiles and with major adverse cardiac events. Men taking ADT for prostate cancer have an increased risk of obesity, insulin resistance, and dyslipidemia [23]. Although a large meta-analysis of randomized controlled trials failed to demonstrate an increased risk of cardiovascular death among men randomized to ADT [24], several retrospective analyses of $\mathrm{ADT}$ in nontrial settings have found increased risks of fatal and nonfatal cardiovascular events, including myocardial infarction, stroke, and peripheral arterial disease $[25,26]$. The use of this therapy in men with preexisting cardiovascular disease is associated with a particularly high risk of events [27-29]. In our cohort, this accounted for $25 \%$ of the study population.

An earlier study done at our centre found that men receiving $\mathrm{ADT}$ had a lower prevalence of cardiovascular disease and risk factors than men who did not receive ADT [30], suggesting that this therapy was being withheld from the highest risk patients, thereby identifying a significant treatment bias. Indeed, men with prostate cancer are twice as likely to die of cardiovascular disease as they are to die of prostate cancer [31]; cardiovascular disease is the leading cause of death in this population. This therefore represents a vulnerable population in whom effective interventions for the primary and secondary prevention of cardiovascular disease are likely to be of high yield. Patients receiving ADT, in whom the risk is expected to further increase with cancer treatment, warrant particular attention in order to minimize iatrogenic escalation of cardiovascular risk. Conversely, effective modification of cardiovascular risk factors to achieve guidelinerecommended targets may result in a greater proportion of prostate cancer patients being deemed eligible for ADT, resulting in improved oncologic outcomes.

In our study cohort, large proportions of patients had not had blood pressure or lipid measurements done prior to initiation of ADT. Current European Association of Urology guidelines recommend that existing general population screening and treatment strategies should be applied to patients receiving ADT [32]. Canadian Cardiovascular Society guidelines recommend annual screening of lipids for individuals with calculated Framingham risks of $\geq 5 \%$ [33], and the Canadian Hypertension Education Program recommends that all adults should be screened for hypertension at all appropriate visits [34]. These guidelines have also addressed pharmacologic and nonpharmacologic strategies for risk factor modification. While management of these risk factors may fall outside the scope of typical oncology practice, cardiooncology clinics can offer multidisciplinary approaches to risk reduction. Cardiooncology clinics focus on prevention and management of cardiovascular disease in cancer patients and aim to remove cardiovascular disease as a barrier to effective cancer treatment [35]. An important aspect of this care is the management of cardiovascular risk factors. In addition, supervised exercise programs offer additional opportunities for risk reduction through physical activity and have been shown to improve cardiovascular risk profiles and cardiopulmonary fitness in prostate cancer patients [36-38].

Very few patients in our cohort had undergone any form of diagnostic testing for CAD. Although the resting ECG is an insensitive method of screening for silent CAD, abnormalities including Q-waves, ST segment depression, and bundle branch block have reported specificities of $>95 \%$ for the prediction of cardiovascular mortality [39]. In our cohort, 37\% of ECGs performed were abnormal, reflecting the higher prevalence of cardiovascular disease in our study cohort than in the population-based cohorts from which sensitivity and specificity analyses are derived [40]. Resting ECG may therefore be a useful screening test in this high-risk population.

Exercise stress testing is a far more sensitive method of screening for CAD than resting electrocardiography. Henriksson and Johansson reported that ischemic changes during an exercise stress test, in conjunction with abnormalities of blood lipids and hormone levels, were strongly predictive of cardiovascular events among men receiving estrogen therapy for prostate cancer [41]. To our knowledge, a similar study has not been performed in men receiving $\mathrm{ADT}$, but the predictive value of exercise testing in the general population has been validated and is widely accepted [42]. Moreover, Wall and colleagues have reported that maximal exercise testing is feasible and safe in men receiving ADT for prostate cancer and have emphasized the importance of exercise testing among men receiving $\mathrm{ADT}$ in the context of additional risk factors who are embarking on an exercise program [43].

A strength of our study was the detailed chart review methodology, likely yielding a higher prevalence of comorbidities and higher rate of cardiac testing than would have been achievable with administrative data or with self-report. There are also limitations to our study. Our sample size was relatively small, potentially limiting the precision of our results. However, we included all patients meeting our inclusion criteria over an entire year and we believe that our sample was representative of the population seen at our centre. In addition, as ADT may be withheld from patients at the highest risk for cardiovascular complications, our results cannot be generalized to all prostate cancer patients. As discussed above, we have reason to believe that the broader prostate cancer population carries, if anything, a greater risk than our cohort [30]. For comparison purposes, we were only able to obtain local population data on the prevalence of hypertension and diabetes, and we were therefore unable to compare the prevalence of cardiovascular disease or dyslipidemia in our cohort to the general population. Our findings, however, were consistent with other epidemiologic studies that have identified associations between cardiovascular risk factors and prostate cancer risk, and the fact that we were able to illustrate the increased prevalence of even a single risk factor highlights the increased risk that may be present in this population. Finally, many of our subjects were missing the necessary data to accurately calculate an FRS. However, our use of zero scores for missing data points has resulted in a 
"minimal estimate" of risk, so we may be confident that we have not overestimated the risk of our cohort.

In conclusion, the results of the present study indicate that despite a low overall comorbidity burden, established cardiovascular disease and cardiovascular risk factors are common among men receiving ADT for intermediate- and high-risk localized prostate cancer. Certain risk factors are more common among prostate cancer patients referred for ADT than among population controls, possibly reflecting the fact that many cardiovascular risk factors are associated with increased prostate cancer risk and highlighting the particularly high-risk nature of this population. Moreover, our findings suggest that suboptimal risk stratification occurs in this population, and accordingly, suboptimal risk modification may result. As cardiovascular disease is a leading cause of death in men with prostate cancer and given that ADT is associated with increased risk of fatal and nonfatal cardiovascular events, this population is likely to benefit from aggressive primary and secondary prevention therapies. The findings of the present study identify an important care gap and an opportunity to improve survivorship care in this population. We propose that care processes to identify atrisk individuals, including standard cardiovascular risk factor assessment and modification or referral to a cardiologyoncology clinic where available, be broadly applied to this patient population to improve patient specific cardiac and cancer related outcomes.

\section{Disclosure}

Tom Pickles has a consultant or advisory role with Astellas Pharma and has received honoraria from Abbott Labs, Ferring Pharma, and Janssen.

\section{Conflict of Interests}

The authors declare that there is no conflict of interests regarding the publication of this paper.

\section{Acknowledgments}

Scott Tyldesley's research time is supported by an investigator award from the Michael Smith Foundation for Cancer Research. He has also received funding from Astellas Pharma, Janssen, and Ferring. Margot K. Davis is supported by a Vancouver Coastal Health Research Institute Mentored Clinician Scientist Award.

\section{References}

[1] R. Siegel, J. Ma, Z. Zou, and A. Jemal, "Cancer statistics, 2014," CA Cancer Journal for Clinicians, vol. 64, no. 1, pp. 9-29, 2014.

[2] Y. Fradet, L. Klotz, J. Trachtenberg, and A. Zlotta, "The burden of prostate cancer in Canada," Journal of the Canadian Urological Association, vol. 3, supplement 2, no. 3, pp. S92-S100, 2009.

[3] A. Collier, S. Ghosh, B. McGlynn, and G. Hollins, "Prostate cancer, androgen deprivation therapy, obesity, the metabolic syndrome, type 2 diabetes, and cardiovascular disease: a review,"
The American Journal of Clinical Oncology: Cancer Clinical Trials, vol. 35, no. 5, pp. 504-509, 2012.

[4] S. M. H. Alibhai, M. Duong-Hua, R. Sutradhar et al., "Impact of androgen deprivation therapy on cardiovascular disease and diabetes," Journal of Clinical Oncology, vol. 27, no. 21, pp. 34523458, 2009.

[5] N. L. Keating, A. J. O’Malley, S. J. Freedland, and M. R. Smith, "Diabetes and cardiovascular disease during androgen deprivation therapy: observational study of veterans with prostate cancer," Journal of the National Cancer Institute, vol. 102, no. 1, pp. 39-46, 2010.

[6] J. A. Tayek, D. Heber, L. O. Byerley, B. Steiner, J. Rajfer, and R. S. Swerdloff, "Nutritional and metabolic effects of gonadotropin-releasing hormone agonist treatment for prostate cancer," Metabolism: Clinical and Experimental, vol. 39, no. 12, pp. 1314-1319, 1990.

[7] J. C. Smith, S. Bennett, L. M. Evans et al., "The effects of induced hypogonadism on arterial stiffness, body composition, and metabolic parameters in males with prostate cancer," Journal of Clinical Endocrinology and Metabolism, vol. 86, no. 9, pp. 42614267, 2001.

[8] C. S. Saigal, J. L. Gore, T. L. Krupski, J. Hanley, M. Schonlau, and M. S. Litwin, "Androgen deprivation therapy increases cardiovascular morbidity in men with prostate cancer," Cancer, vol. 110, no. 7, pp. 1493-1500, 2007.

[9] M. van Hemelrijck, H. Garmo, L. Holmberg, P. Stattin, and J. Adolfsson, "Multiple events of fractures and cardiovascular and thromboembolic disease following prostate cancer diagnosis: results from the population-based PCBaSe Sweden," European Urology, vol. 61, no. 4, pp. 690-700, 2012.

[10] J. A. Efstathiou, K. Bae, W. U. Shipley et al., "Cardiovascular mortality and duration of androgen deprivation for locally advanced prostate cancer: analysis of RTOG 92-02," European Urology, vol. 54, no. 4, pp. 816-824, 2008.

[11] K. E. Weaver, R. E. Foraker, C. M. Alfano et al., "Cardiovascular risk factors among long-term survivors of breast, prostate, colorectal, and gynecologic cancers: a gap in survivorship care?" Journal of Cancer Survivorship, vol. 7, no. 2, pp. 253-261, 2013.

[12] P. W. F. Wilson, R. B. D’Agostino, D. Levy, A. M. Belanger, H. Silbershatz, and W. B. Kannel, "Prediction of coronary heart disease using risk factor categories," Circulation, vol. 97, no. 18, pp. 1837-1847, 1998.

[13] H. Quan, B. Li, C. M. Couris et al., "Updating and validating the charlson comorbidity index and score for risk adjustment in hospital discharge abstracts using data from 6 countries," American Journal of Epidemiology, vol. 173, no. 6, pp. 676-682, 2011.

[14] Statistics Canada, Canadian Community Health Survey (CCHS), 2012, http://www5.statcan.gc.ca.

[15] K. Esposito, P. Chiodini, A. Capuano et al., "Effect ofmetabolic syndrome and its components on prostate cancer risk:Metaanalysis," Journal of Endocrinological Investigation, vol. 36, no. 2, pp. 132-139, 2013.

[16] R. M. Martin, L. Vatten, D. Gunnell, and P. Romundstad, "Blood pressure and risk of prostate cancer: cohort Norway (CONOR)," Cancer Causes and Control, vol. 21, no. 3, pp. 463-472, 2010.

[17] C. Pelucchi, D. Serraino, E. Negri et al., "The metabolic syndrome and risk of prostate cancer in Italy," Annals of Epidemiology, vol. 21, no. 11, pp. 835-841, 2011.

[18] K. N. Sourbeer, L. E. Howard, G. L. Andriole et al., "Metabolic syndrome-like components and prostate cancer risk: results 
from the Reduction by Dutasteride of Prostate Cancer Events (REDUCE) study," BJU International, 2014.

[19] J.-A. Thomas II, L. Gerber, L. L. Bañez et al., "Prostate cancer risk in men with baseline history of coronary artery disease: results from the reduce study," Cancer Epidemiology Biomarkers and Prevention, vol. 21, no. 4, pp. 576-581, 2012.

[20] J. M. Post, J. L. Beebe-Dimmer, H. Morgenstern et al., "The metabolic syndrome and biochemical recurrence following radical prostatectomy," Prostate Cancer, vol. 2011, Article ID 245642, 6 pages, 2011.

[21] R. Asmar, J. L. Beebe-Dimmer, K. Korgavkar, G. R. Keele, and K. A. Cooney, "Hypertension, obesity and prostate cancer biochemical recurrence after radical prostatectomy," Prostate cancer and Prostatic Diseases, vol. 16, no. 1, pp. 62-66, 2013.

[22] M. Shiota, A. Yokomizo, A. Takeuchi et al., "The feature of metabolic syndrome is a risk factor for biochemical recurrence after radical prostatectomy," Journal of Surgical Oncology, vol. 110, no. 4, pp. 476-481, 2014.

[23] P. J. Saylor and M. R. Smith, "Metabolic complications of androgen deprivation therapy for prostate cancer," The Journal of Urology, vol. 189, no. 1, supplement, pp. S34-S44, 2013.

[24] P. L. Nguyen, Y. Je, F. A. B. Schutz et al., "Association of androgen deprivation therapy with cardiovascular death in patients with prostate cancer: a meta-analysis of randomized trials," The Journal of the American Medical Association, vol. 306, no. 21, pp. 2359-2366, 2011.

[25] C. G. Jespersen, M. Nørgaard, and M. Borre, "Androgendeprivation therapy in treatment of prostate cancer and risk of myocardial infarction and stroke: a nationwide Danish population-based cohort study," European Urology, vol. 65, no. 4, pp. 704-709, 2014.

[26] J. C. Hu, S. B. Williams, A. J. O’Malley, M. R. Smith, P. L. Nguyen, and N. L. Keating, "Androgen-deprivation therapy for nonmetastatic prostate cancer is associated with an increased risk of peripheral arterial disease and venous thromboembolism," European Urology, vol. 61, no. 6, pp. 1119-1128, 2012.

[27] P. L. Nguyen, M. H. Chen, S. Z. Goldhaber et al., "Coronary revascularization and mortality in men with congestive heart failure or prior myocardial infarction who receive androgen deprivation," Cancer, vol. 117, no. 2, pp. 406-413, 2011.

[28] D. R. Ziehr, M.-H. Chen, D. Zhang et al., "Association of androgen-deprivation therapy with excess cardiac-specific mortality in men with prostate cancer," BJU International, 2014.

[29] A. Nanda, M.-H. Chen, M. H. Braccioforte, B. J. Moran, and A. V. D’Amico, "Hormonal therapy use for prostate cancer and mortality in men with coronary artery disease-induced congestive heart failure or myocardial infarction," The Journal of the American Medical Association, vol. 302, no. 8, pp. 866873, 2009.

[30] J. Kim, M. Vaid, S. Tyldesley, R. Woods, and T. Pickles, "Population-based study of cardiovascular mortality among patients with prostate cancer treated with radical external beam radiation therapy with and without adjuvant androgen deprivation therapy at the British Columbia cancer agency," International Journal of Radiation Oncology Biology Physics, vol. 80, no. 3, pp. 742-750, 2011.

[31] S. Shikanov, M. Kocherginsky, A. L. Shalhav, and S. E. Eggener, "Cause-specific mortality following radical prostatectomy," Prostate Cancer and Prostatic Diseases, vol. 15, no. 1, pp. 106-110, 2012.

[32] A. Heidenreich, P. J. Bastian, J. Bellmunt et al., "EAU guidelines on prostate cancer. Part II: treatment of advanced, relapsing, and castration-resistant prostate cancer," European Urology, vol. 65, no. 2, pp. 467-479, 2014.

[33] T. J. Anderson, J. Grégoire, R. A. Hegele et al., "Update of the Canadian Cardiovascular Society guidelines for the diagnosis and treatment of dyslipidemia for the prevention of cardiovascular disease in the adult," Canadian Journal of Cardiology, vol. 29, no. 2, pp. 151-167, 2013.

[34] D. G. Hackam, R. R. Quinn, P. Ravani et al., “The 2013 Canadian Hypertension Education Program recommendations for blood pressure measurement, diagnosis, assessment of risk, prevention, and treatment of hypertension," Canadian Journal of Cardiology, vol. 29, no. 5, pp. 528-542, 2013.

[35] D. J. Lenihan, D. Cardinale, and C. M. Cipolla, “The compelling need for a cardiology and oncology partnership and the birth of the international cardioncology society," Progress in Cardiovascular Diseases, vol. 53, no. 2, pp. 88-93, 2010.

[36] N. Beydoun, J. A. Bucci, Y. S. Chin, N. Spry, R. Newton, and D. A. Galvão, "Prospective study of exercise intervention in prostate cancer patients on androgen deprivation therapy," Journal of Medical Imaging and Radiation Oncology, vol. 58, no. 3, pp. 369-376, 2014.

[37] D. A. Galvão, N. Spry, J. Denham et al., "A multicentre yearlong randomised controlled trial of exercise training targeting physical functioning in men with prostate cancer previously treated with androgen suppression and radiation from TROG 03.04 RADAR," European Urology, vol. 65, no. 5, pp. 856-864, 2014.

[38] F. T. Baumann, E. M. Zopf, and W. Bloch, "Clinical exercise interventions in prostate cancer patients-a systematic review of randomized controlled trials," Supportive Care in Cancer, vol. 20, no. 2, pp. 221-233, 2012.

[39] E. A. Ashley, V. K. Raxwal, and V. F. Froelicher, "The prevalence and prognostic significance of electrocardiographic abnormalities," Current Problems in Cardiology, vol. 25, no. 1, pp. 1-72, 2000.

[40] W. B. Kannel, T. Gordon, and D. Offutt, "Left ventricular hypertrophy by electrocardiogram. Prevalence, incidence, and mortality in the Framingham study," Annals of Internal Medicine, vol. 71, no. 1, pp. 89-105, 1969.

[41] P. Henriksson and S.-E. Johansson, "Prediction of cardiovascular complications in patients with prostatic cancer treated with estrogen," The American Journal of Epidemiology, vol. 125, no. 6, pp. 970-978, 1987.

[42] D. B. Mark, L. Shaw, F. E. Harrell et al., "Prognostic value of a treadmill exercise score in outpatients with suspected coronary artery disease," The New England Journal of Medicine, vol. 325, no. 12, pp. 849-853, 1991.

[43] B. A. Wall, D. A. Galvão, N. Fatehee et al., "Maximal exercise testing of men with prostate cancer being treated with androgen deprivation therapy," Medicine \& Science in Sports \& Exercise, vol. 46, no. 12, pp. 2210-2215, 2014. 


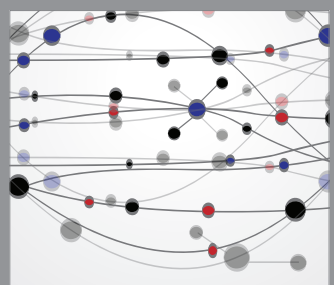

The Scientific World Journal
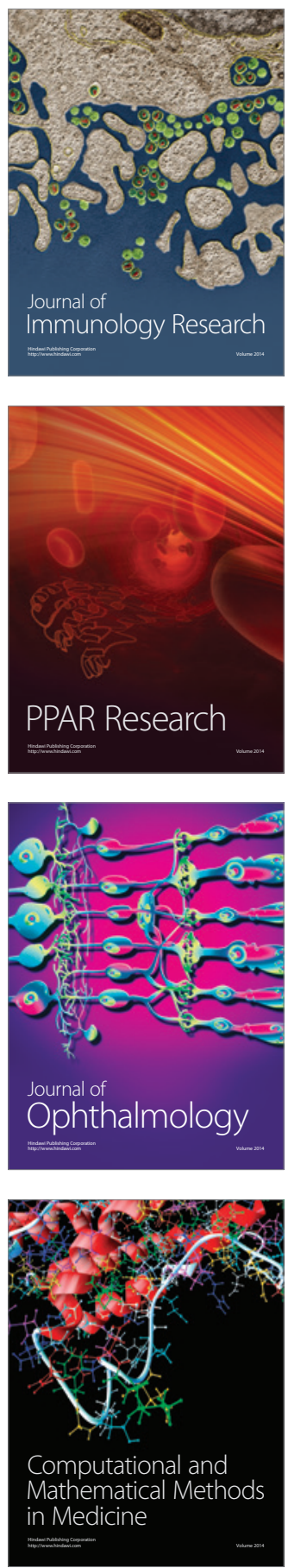

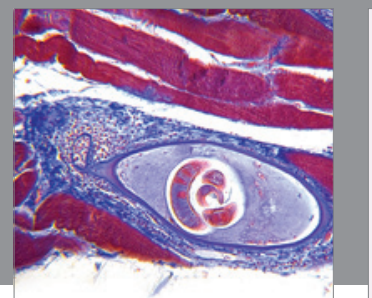

Gastroenterology

Research and Practice
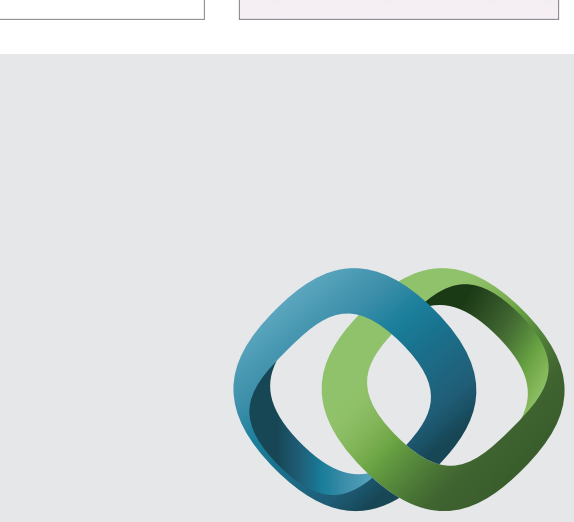

\section{Hindawi}

Submit your manuscripts at

http://www.hindawi.com
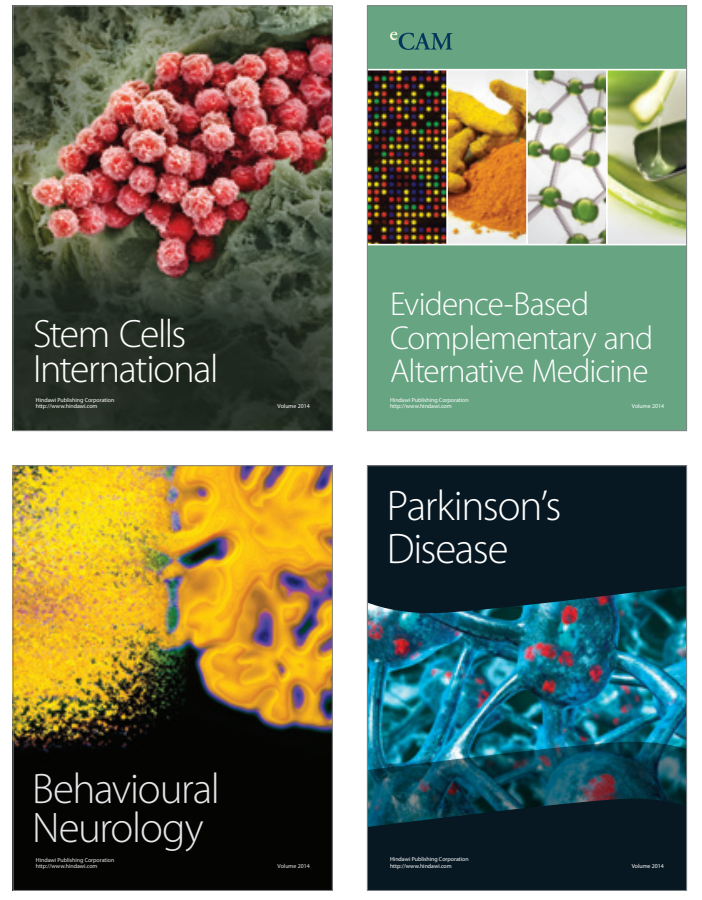
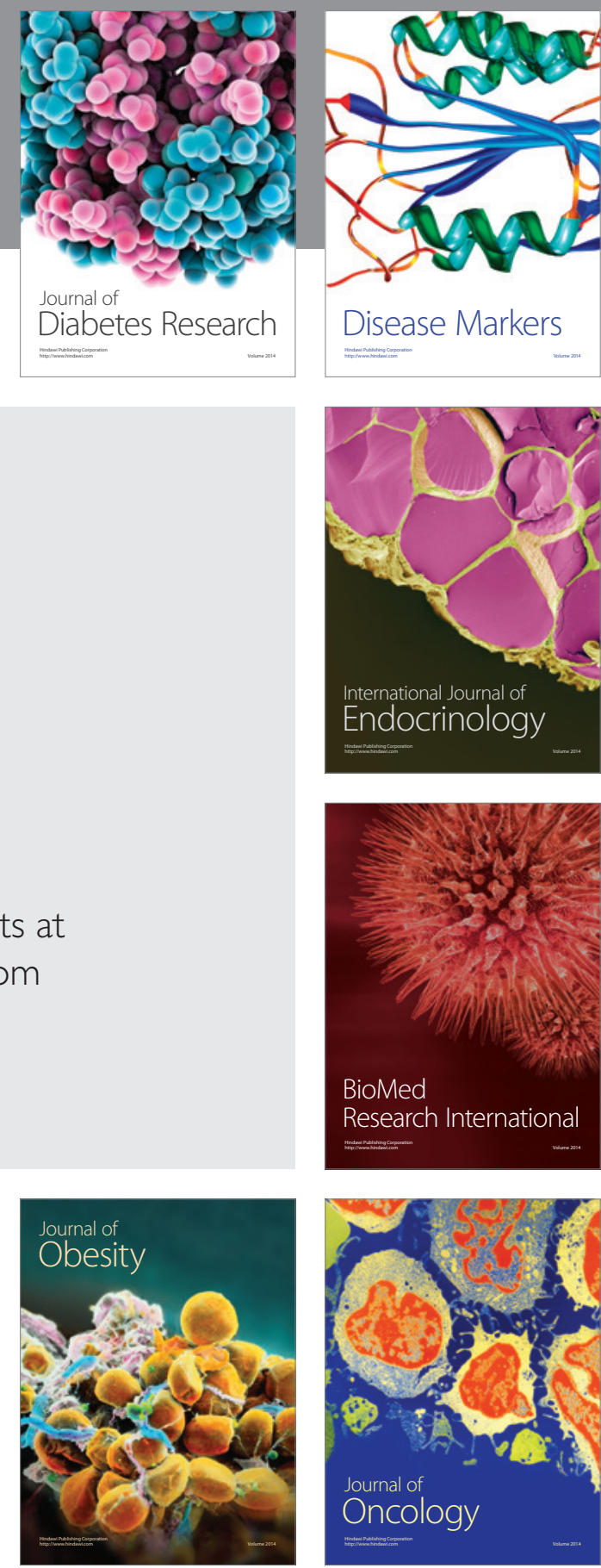

Disease Markers
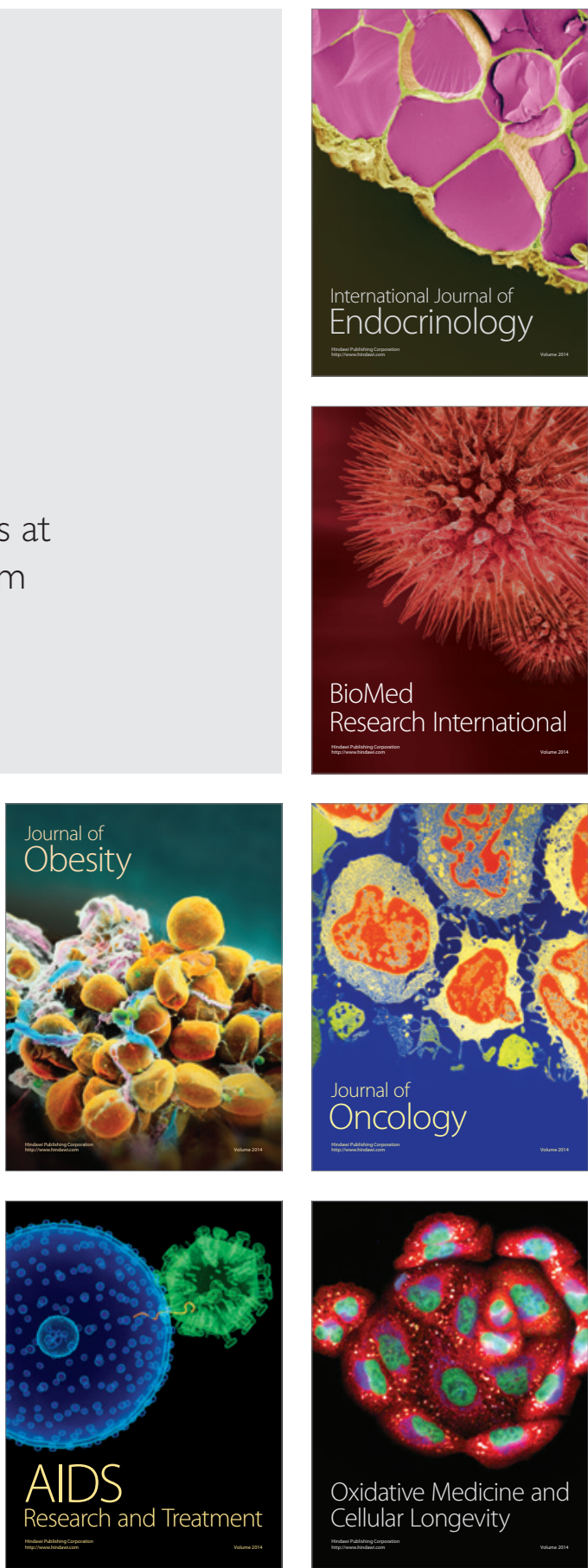